\title{
Necrotizing Fasciitis in the Puerperium of a Woman with Complement Deficiency: Case Report and Review Literature
}

\author{
Janete Vettorazzi1,2,3,4*, Edimárlei Gonsales Valério1,2,3,4, Gustavo Adolpho Moreira Faulhaber², \\ Amanda Vilaverde Perez ${ }^{3}$, Mariana Sbaraini'3 ${ }^{3}$ Daniela Vanessa Vettori ${ }^{1,4}$ \\ ${ }^{1}$ Postgraduation Program in Health Sciences: Gynecology and Obstetrics, Universidade Federal do Rio Grande do Sul (UFRGS), \\ Porto Alegre, Brazil \\ ${ }^{2}$ Department of Gynecology and Obstetrics, Faculty of Medicine (FAMED), Universidade Federal do Rio Grande do Sul, Porto \\ Alegre, Brazil \\ ${ }^{3}$ Faculty of Medicine (FAMED), Universidade Federal do Rio Grande do Sul, Porto Alegre, Brazil \\ ${ }^{4}$ Service of Obstetrics and Gynecology, Hospital de Clínicas de Porto Alegre (HCPA), Porto Alegre, Brazil \\ Email: ^jvettorazzi@hcpa.edu.br, edimarleigv@terra.com.br, gfaulhaber@hcpa.edu.br, amandavperez@gmail.com, \\ marisbaraini@gmail.com,dvettori@hcpa.edu.br
}

How to cite this paper: Vettorazzi, J., Valério, E.G., Faulhaber, G.A.M., Perez, A.V., Sbaraini, M. and Vettori, D.V. (2018) Necrotizing Fasciitis in the Puerperium of a Woman with Complement Deficiency: Case Report and Review Literature. Open Journal of Obstetrics and Gynecology, 8, 1236-1246.

https://doi.org/10.4236/ojog.2018.812125

Received: September 3, 2018

Accepted: October 23, 2018

Published: October 26, 2018

Copyright $\odot 2018$ by authors and Scientific Research Publishing Inc. This work is licensed under the Creative Commons Attribution International License (CC BY 4.0).

http://creativecommons.org/licenses/by/4.0/

\section{(c) (i) Open Access}

\begin{abstract}
Complement deficiencies are uncommon types of primary immunodeficiency. Necrotizing fasciitis is a rare complication in pregnancy characterized by soft tissue invasion and necrosis of the subcutaneous and other adjacent tissues, leading to high mortality rates. We report a case of a 29 -year-old pregnant woman with functional deficiency of the $\mathrm{C} 4$ complement component and short uterine cervix. Admitted at the hospital with preterm labor, she received multiple doses of immunoglobulin. After 8 weeks, she had a premature membrane rupture, and due to pelvic presentation she had a cesarean. The patient presented multiple obstetric complications, such as operative wound infection, endometritis, sepsis, necrotizing fasciitis and pelvic septic thrombophlebitis. She underwent multiple antimicrobial schemes, a hysterectomy and 4 extensive debridements of the abdominal wall because of significant necrosis. She stayed at the hospital for 101 days (32 of those in ICU in immediate postpartum). 41 days after cesarean, patient was discharged in good conditions. Our case emphasizes individual handling and high multiple doses of immunoglobulin for favorable outcome of the case.
\end{abstract}

\section{Keywords}

Pregnancy, Immunodeficiency, C4 Factor, C4 Deficiency, Necrotizing 
Fasciitis, Puerperal Infection

\section{Introduction}

The complement system plays an important role in the innate and adaptive immune response against pathogens. It is essential for removing damaged cells, avoiding infections by enhancing the ability of clearing microorganisms and helping the modulation of the immune response [1]. Complement deficiencies are rare, with a worldwide prevalence of $0.03 \%$ [2], and predispose bacterial infection and/or autoimmune diseases. Individuals with abnormalities in the early components of the complement (especially C1q, C2 and C4) have a minimum risk of infection but a high risk of developing autoimmune diseases such as systemic lupus erythematosus (SLE) and rheumatoid arthritis. Increased susceptibility to these diseases is related to the inefficient removal of immune complexes [3], and C4, part of the classical complement pathway, is closely linked to the removal of these complexes and of apoptotic debris [4].

In patients with $\mathrm{C} 4$ deficiency, the main treatment is based in the use of immunoglobulin, which helps reducing the frequency and severity of infections. Intravenous preparations start with $400-600 \mathrm{mg} / \mathrm{kg}$ every 3 to 4 weeks and are almost exclusively made of IgG [5] [6]. Monitoring of IgG levels should be performed every 3 months and should remain above $500 \mathrm{mg} / \mathrm{dL}$. Most adverse effects are related to infusion rate, most commonly being fever, tremors, arthralgia, myalgia, abdominal pain, nausea and headache. Fatal events are rare and can be prevented by careful medical supervision and early management [5] [6].

We report a case of a pregnant woman with $\mathrm{C} 4$ deficiency with multiple previous gestational losses. She progressed during the puerperium to septic pelvic thrombophlebitis and necrotizing fasciitis in the abdominal surgical wound. She had a good evolution and was discharged without sequelae. This case involves two rare pathologies, without previous reports in literature of simultaneous occurrence and survival: immunodeficiencies of complement factors and necrotizing fasciitis. In reporting the case, we intend to help management of similar cases that may occur. The case occurred in the Hospital de Clínicas de Porto Alegre, in the state of Rio Grande do Sul, Brazil-a public-private institution linked to the Universidade Federal do Rio Grande do Sul.

\section{Case Report}

Patient has consented to the disclosure of her case for academic and scientific purposes. Pregnant woman, 29 years old, white. Obstetric history of six pregnancies-two vaginal deliveries and three abortions. The two vaginal deliveries had neonatal death, weighting 498 grams (gestational age of 22 weeks) and 650 grams (gestacional age of 25 weeks). In both cases, she was hospitalized after 
rupture of the membranes and labor was induced. The abortions occurred in the first pregnancy trimester. Cerclage was performed in the third and fourth gestation, however they both evolved with ovular infection and gestational loss after two weeks. The patient hadn't used immunoglobulin in any previous gestation. She had a sister with history of multiple gestational losses and a father which died of sepsis after thoracic trauma. Both of them had C4 deficiency.

First prenatal consult happened at 15 weeks, when progesterone $200 \mathrm{mg} /$ day was prescribed and serial measurements of the uterine cervix by transvaginal ultrasound were programmed. The uterine cervix was measured biweekly until 22 weeks, being initially $37 \mathrm{~mm}$ and progressing to $24 \mathrm{~mm}$, when Ingamed ${ }^{\circledR}$ cervical pessary was inserted.

The patient was hospitalized at 21 weeks due to a vaginal infection, receiving clindamycin, azithromycin and metronidazole, Use of vaginal progesterone was maintained and a 5-day pulse of intravenous immunoglobulin was initiated. The patient's evolution followed as described in the table below.

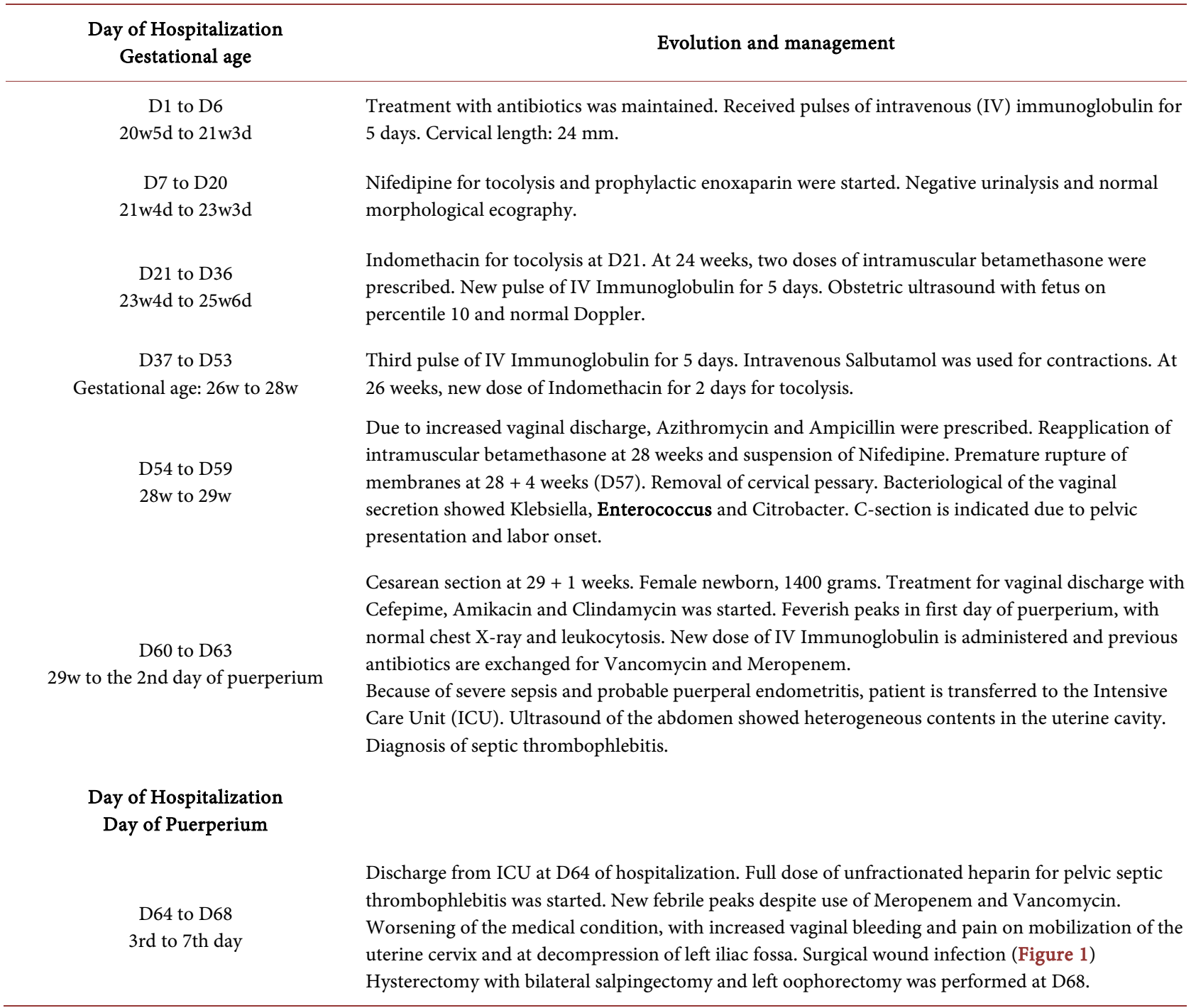




\section{Continued}

D71 to D75

9 th to $13 \mathrm{rd}$

D76 to D79

13 th to 16 th

D80 to D86

17 th to 22 th

D87 to D100

23rd to 40 th
Use of IV Immunoglobulin and full anticoagulation with unfractionated heparin infusion pump. New fever peak in the first postoperative day, operative wound with hyperemia and drainage of serohematic secretion. Amikacin was added to the scheme with Vancomycin and Meropenem. Patient continues to be feverish and leukocytosis worsens $(33,000)$. Abdominal tomography showed signs of thrombosis in the right gonadal vein, grouped fluid collections with gaseous bubbles permeating next to the infraumbilical anterior abdominal wall musculature, and small accumulation of fluid and densification of adipose planes in the pelvis. Operative wound evolved with necrosis, crepitation and fluctuation. Amikacin is suspended and Polymyxin B is initiated for necrotizing fasciitis.

First debridement of the operative wound, with purulent discharge and muscular involvement (Figure 2). Negative culture of abdominal wall necrosis for coagulase-negative Staphylococcus. Patient stays at ICU in the postoperative period. Polymyxin B, Clindamycin, Vancomycin and Meropenem were maintained, and unfractionated heparin was reinstated. New febrile peak at D6 post hysterectomy, elevation of C-reactive protein and leukocytosis. Operative wound with blisters, exudate and fibrin. Fluconazole is initiated. New worsening of the medical state.

New debridement (Figure 3) of operative wound in D9 post hysterectomy showed bullous areas at its borders, rectus abdominis muscle with infiltration, areas of purulent suppuration and accumulation of devitalized tissue at the lower edge of the wound. Retraction of left rectum muscle and removal of left bullous areas was performed until viable tissue was found. Fluconazole, Clindamycin, Polymyxin B, Meropenem and Vancomycin maintained. Intubation is necessary at D11 post hysterectomy. Worsening of the suprapubic and lateral infiltrate of the operative wound, with extension to the left iliac fossa and inguinal region, where areas of necrosis with blisters were found. New febrile peak and worsening of renal function. A dose of immunoglobulin and a new debridement in postoperative D11 were prescribed.

Patient returned from debridement in severe septic shock. She was in mechanical ventilation requiring increasing doses of vasopressin and hydrocortisone. Hemodialysis was started. In postoperative D13, new debridement of surgical wound and vacuum dressing exchange. Patient showed hemodynamic improvement, when sedation was paused and vasopressors were suspended. Hemodialysis stopped at D18 after hysterectomy.

Patient is discharged from ICU at postoperative D25, hemodynamically stable and without vasoactive drugs. No signs of sepsis or organic dysfunction were found, and renal function was preserved. She was discharged from the hospital in postoperative D32 (D100 of hospitalization) using warfarin but without antibiotics. Her skin color was modified, with a darker shade than previously (Figure 4).

Patient was discharged in good conditions.

W: weeks; D: days; IV: intravenous; ICU: Intensive Care Unit.

The patient used warfarin for three months and after four months the color of the skin returned to its usual color and the surgical scar was in the final stage of healing (Figure 5 \& Figure 6). After 1 year, she returned without complications and her skin color had returned to its normal.

The newborn remained 53 days in the neonatal ICU, requiring mechanical ventilation and a nasogastric tube. He was discharged in good general condition along with the mother. He maintained follow-up visits, evolving without sequelae. Currently, he has a palatal hemangioma in regression.

\section{Discussion}

During pregnancy, the innate and adaptive immune system must be regulated to ensure the survival of the mother and the fetus. The complement system is a part 


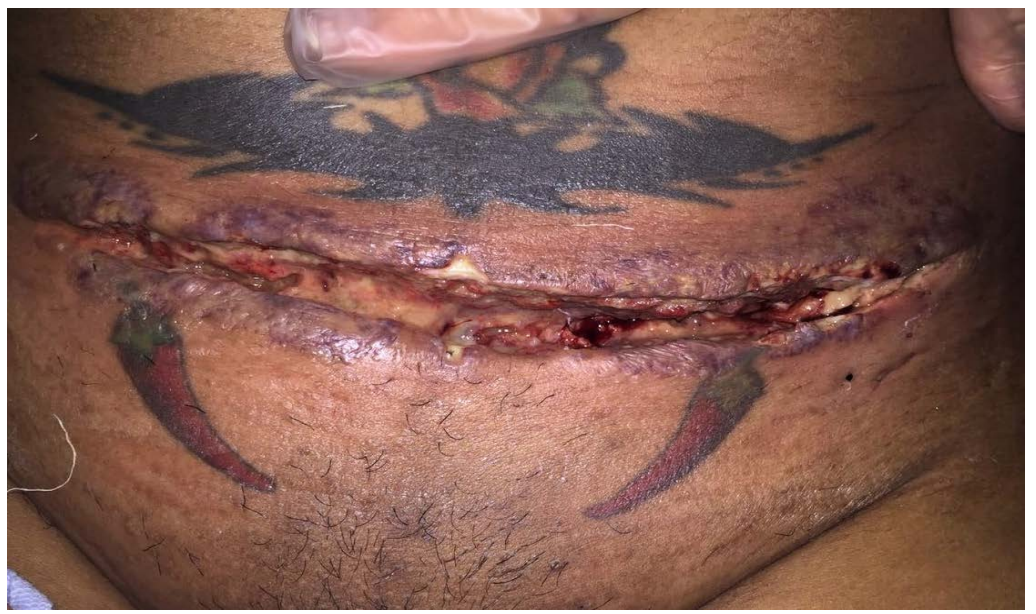

Figure 1. Initial wound infection.

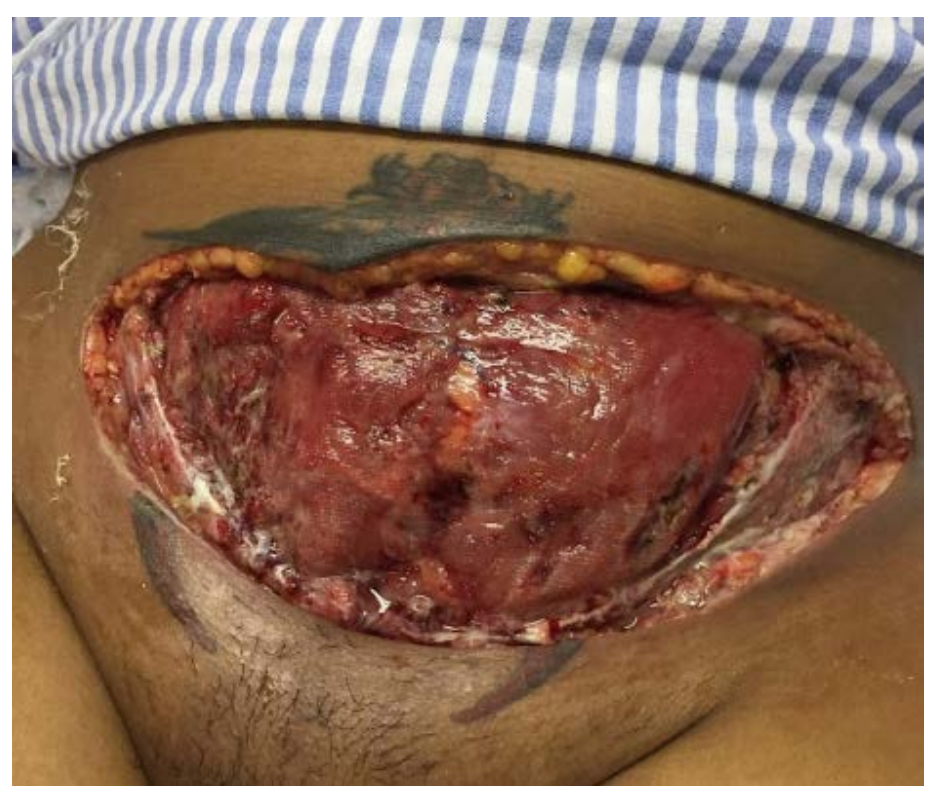

Figure 2. Patient with necrotizing fasciitis after first debridement.

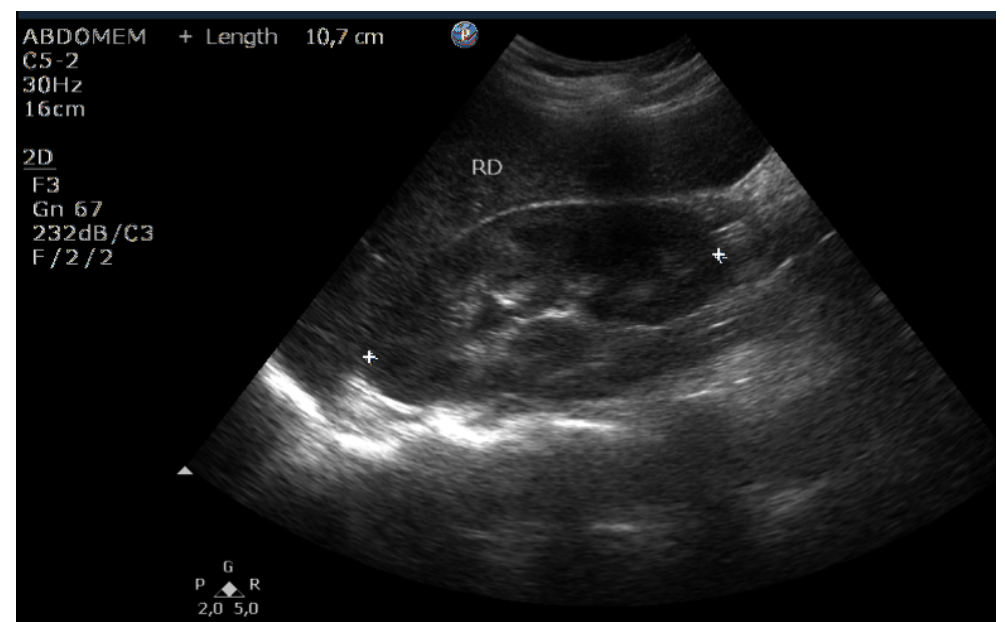

Figure 3. Abdominal ultrasound with heterogeneous contents in the uterine cavity. 


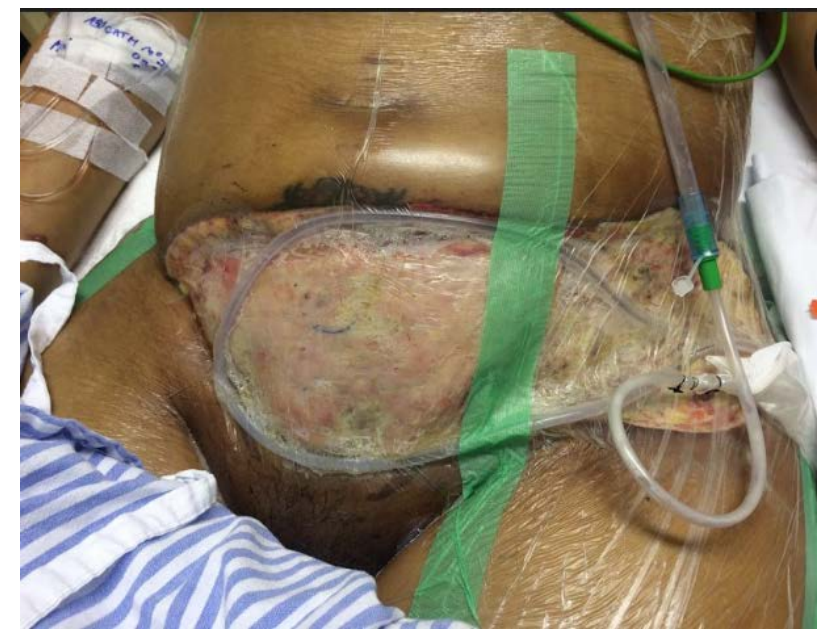

Figure 4. Patient with occlusive dressing after debridement.

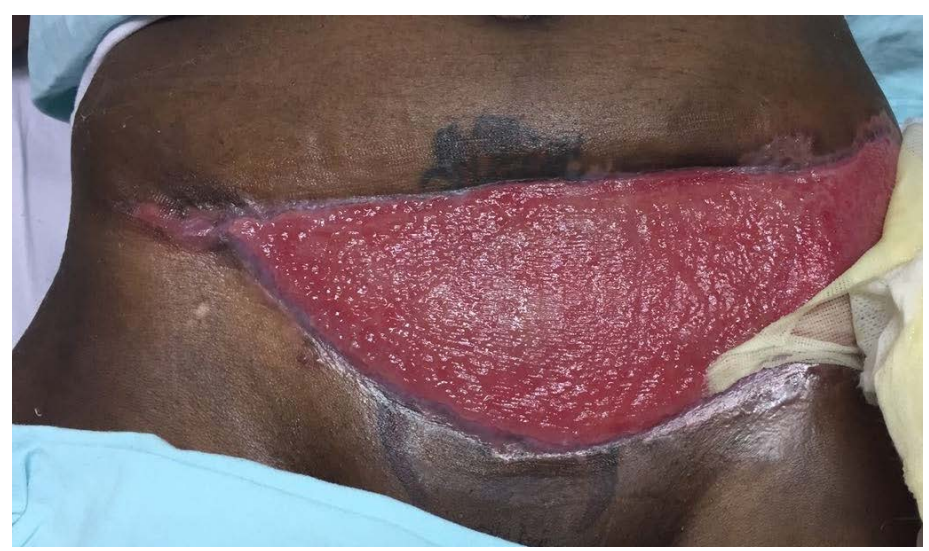

Figure 5. Operative wound with granulation tissue after 40 days of puerperium. Dullness of the patient's color due to the prolonged use of antibiotics.

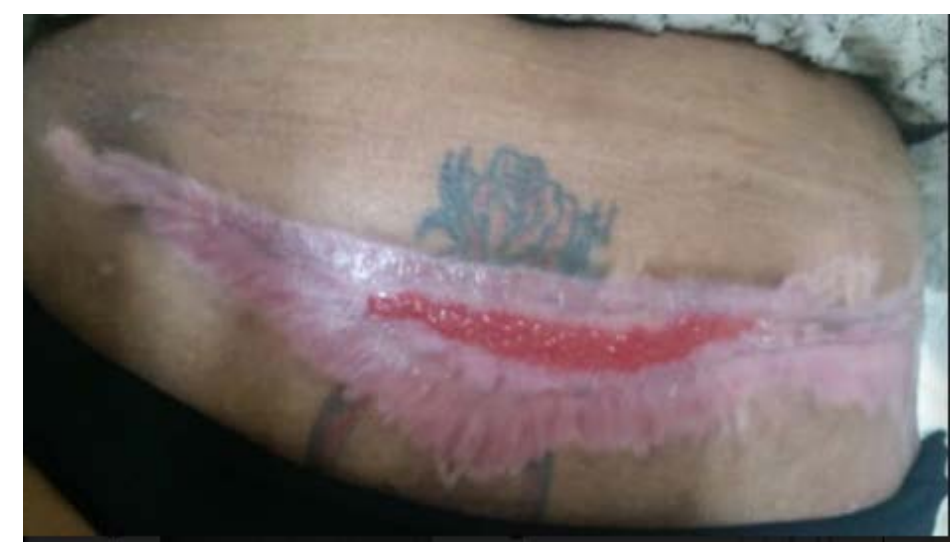

Figure 6. Patient 4 months after surgery. The color of the skin returned to its usual color.

of the immune system that consists of a set of proteins that act sequentially, providing a rapid and powerful defense to the body by identifying and destroying pathogens. Its inadequate activation can cause a number of complications.

More than 50 proteins constitute the complement system, and C4 is part of 
the classical complement route. IgG and IgM antibodies bind to antigens and initiate a cascade of events, where the $\mathrm{C} 1$ factor cleaves $\mathrm{C} 4$ and $\mathrm{C} 2$ in $\mathrm{C} 4 \mathrm{~b}$ and $\mathrm{C} 2 \mathrm{~b}$, respectively. These factors interact and form $\mathrm{C} 3$ convertase, which converts $\mathrm{C} 3$ to $\mathrm{C} 3 \mathrm{a}$ (that produces inflammatory response) and $\mathrm{C} 3 \mathrm{~b}$ (that has an opsonization function). The addition of $\mathrm{C} 3 \mathrm{~b}$ to $\mathrm{C} 3$ convertase generates $\mathrm{C} 5$ convertase, which cleaves $\mathrm{C} 5$ to $\mathrm{C} 5 \mathrm{a}$ and $\mathrm{C} 5 \mathrm{~b}$. C5b then connects to $\mathrm{C} 6$ and $\mathrm{C} 7$, forming a complex that binds to the membrane. The $\mathrm{C} 5 \mathrm{bC} 6 \mathrm{C} 7$ complex binds to $\mathrm{C} 8$ and multiple $\mathrm{C} 9$, forming the membrane attack complex (MAC). This complex forms a channel that allows the free diffusion of substance, disrupting the cellular osmotic balance and leading to death of the invading organism [7].

Inherited complement deficiencies are rare [2], corresponding to $4.9 \%$ of all primary immunodeficiencies [8]. The most common one is $\mathrm{C} 1$ deficiency, occurring in $0.01 \%$ of the population [9]. Severe deficiencies of complement proteins predispose to infections, mainly by encapsulated bacteria such as Streptococcus pneumoniae. In addition, $\mathrm{C} 1, \mathrm{C} 2$, and $\mathrm{C} 4$ deficiencies may predispose to autoimmune diseases, primarily collagen vascular diseases and systemic lupus erythematosus. This is due to failure in removing immune complexes and necrotic material. More than $75 \%$ of patients with $\mathrm{C} 4$ deficiency have some manifestation of autoimmunity [10]. Studies have shown that patients with C4 deficiency have a decrease in regulatory $\mathrm{T}$ cells, which are important to prevent the appearance of aberrant autoimmune responses [11]. Complete homozygosity for $\mathrm{C} 4$ deficiency is rare. The $\mathrm{C} 4$ gene is located on chromosome $6 \mathrm{p} 21.3$, and there are two isotypes of the gene product (C4A and C4B5). Healthy individuals usually have two copies of each of the isotypes, while those with less than four copies of $\mathrm{C} 4$ are more likely to develop autoimmune diseases [4] [12].

Pregnancy alone can increase infection by suffering various mechanical and physiological adaptations and by creating an immunosuppressive state. These changes in a patient with deficiency of complement factors-important in regulating the immune response-play a prominent role in severe infections and subsequent sepsis [13]. Infections are the fifth leading cause of maternal death [14].

Necrotizing fasciitis (NF) is an invasive and potentially lethal soft tissue infection. It is characterized by necrosis of the subcutaneous tissue, superficial fascia and other adjacent tissues. The infection can involve epidermis, dermis, subcutaneous tissue, fascia and muscle. It is considered a surgical emergency, with mortality exceeding 40\% [15] and increasing incidence in recent years [16]. Pregnancy, delivery and gynecological procedures are important risk factors for NF. Other risk factors are those that predispose infections, such as peripheral vascular disease, diabetes mellitus, impaired immune system, and surgical procedures that compromise skin integrity. In a retrospective study with 23 patients with NF in lower abdomen or pelvis, 34.7\% had diabetes [17]. Obesity, which increases the infection risk during pregnancy and cesarean delivery, is also an important risk factor [18]. 
The fasciitis can be divided considering microbiology or presence of gas in the tissues. Polymicrobial fasciitis is caused by anaerobic and aerobic bacteria, with typically at least one isolated anaerobic (Bacteroides, Clostridium or Peptostreptococcus) in combination with enterobacteria (Escherichia coli, Enterobacter, Klebsiella, Proteus). Monomicrobial fasciitis is usually caused by Staphylococcus, or group A and other beta hemolytic Streptococcus. Pure myonecrosis by Clostridium perfringens has a more homogenous muscle invasion and a higher rate of mortality, being the only one that needs to be differentiated from other necrotizing soft tissue infections [4]. The incidence of this type of NF varies from 0.3 to 15 per 100,000 people.

Main manifestations of NF include severe pain, edema, erythema, fever, crepitation and epithelial necrosis. The disease has rapid onset and progression, involving mainly the vulva, perineum, lower limbs and abdominal wall. Skin involvement is usually smaller than the extent of necrosis in the underlying tissue, turning the differentiation between NF and cellulitis difficult [19]. Definitive diagnosis occurs after surgical debridement, with microbiological and histological evaluation of the infected tissues. Imaging findings precede skin involvement, aiding in diagnosis. Magnetic Resonance imaging can determine the extent of inflammation in the fascia and necrosis of infectious tissue [20]. Crepitation, presence of gas in soft tissue or bubble formation in imaging have a good sensitivity for diagnosis [4]. Among laboratory tests, base deficit and blood lactate are often elevated. Without rapid and efficient intervention, NF quickly leads to septic shock, multiple organ failure and death. Most of the time, this illness requires long hospitalization, days in ICU and high hospital expenses [16].

Prognosis in NF depends on rapid diagnosis and aggressive multidisciplinary management, involving deep debridement of all necrotic tissue, intravenous antibiotics, appropriate analgesia and intensive care in ICU [19]. Antibiotics are administered empirically before culture results and can be adjusted later according to the antibiogram. It should cover gram positive cocci, enteric gram negative rods and anaerobics. Daily evaluation of the open wound and surgical debridement until the infection is no longer perceived decreases mortality [15]. Normally, the procedure is repeated every 2 days until there is no evidence of necrotic tissue. Closure of the wounds begins as soon as possible, just when the viable tissue allows the skin to be re-approximated or grafted [19]. Hyperbaric oxygen therapy has been used adjuvantly, and although it does not decrease mortality, it can accelerate wound closure. This technique improves oxygen supply to compromised ischemic tissue, increases phagocytosis of polymorphonuclear cells, acts as a bactericidal agent for anaerobic organisms and can disrupt the production of alpha toxin. However, studies validating this therapy are still lacking [21].

The use of IV Immunoglobulin also appears to be beneficial for the survival of patients with NF [19]. Human Immunoglobulin can be beneficial in primary immunodeficiencies, such as those caused by absence of B cells or inefficient production of antibodies, and primary defects with hypogammaglobulinemia. 
Currently, there is sufficient clinical evidence showing that Immunoglobulin decreases frequency and severity of infections and of hospitalizations in patients with primary immunodeficiencies. However, much of this evidence refers to immunodeficiencies that compromise the production of antibodies (the most prevalent ones). There are no studies showing benefits of using IV Immunoglobulin in patients with complement deficiency; however, the lack of other treatments for this disease and the good results in other immunodeficiencies justify its use in patients with $\mathrm{C} 4$ deficiency.

The appropriate dose of immunoglobulin for primary immunodeficiencies is still being studied. An initial dose of $400-600 \mathrm{mg} / \mathrm{kg}$ is advised every 3 to 4 weeks. The frequency of adverse effects ranges from $0.6 \%$ to $30 \%$, being higher in the presence of infections or at the first infusion [22]. This is due to the formation of antigen-antibody complexes, which can be reduced if the patient is afebrile or using antimicrobials. To reduce adverse effects, infusion should be slow and by infusion pump. During the infusion, most common symptoms include tremors and fevers (mimicking infection), abdominal pain, nausea and headache. In these cases, the infusion should be discontinued and the symptoms managed.

In necrotizing fasciitis, IV Immunoglobulin also appears to be beneficial. It is supposedly able to inhibit the mitogenic activity of bacteria, especially gram positive. In addition, Immunoglobulin can block $\mathrm{T}$ lymphocytes activation by bacterial antigens, decreasing the production of inflammatory cytokines [23] [24].

\section{Conclusion}

Complement deficiencies are extremely rare conditions that are associated with the development of autoimmune diseases. In pregnancy, an immunosuppressive state alone, they can cause numerous adverse outcomes. Necrotizing fasciitis is an infection that has immunosuppressive states as an important risk factor. The reported case shows how immunocompromised pregnant women need follow-up in high-risk prenatal care and, if possible, the use of prophylactic IV Immunoglobulin. In cases of infection, rapid diagnosis and multidisciplinary therapy, with a surgical approach and use of antibiotics, are important. We emphasize the importance of immunoglobulin use for the patient's excellent outcome and the relevance of knowing family history and efficacy of immunoglobulin use in other relatives. A clear understanding of the role of the complement system in pregnancy - and its possible deficiencies-is necessary to prevent adverse outcomes. No cases of patients with both necrotizing fasciitis and $\mathrm{C} 4 \mathrm{im}$ munodeficiency have survived. We believe that future cases may benefit from the successful management of this patient using immunoglobulin.

\section{Acknowledgements}

We would like to express our deepest gratitude to the participants for their time and patience throughout this study. 


\section{Financial Disclosure}

The authors have no financial relationships relevant to this article to disclose.

\section{Conflicts of Interest}

The authors have no conflicts of interest to disclose.

\section{References}

[1] Macedo, A.C.L. and Isaac, L. (2016) Systemic Lupus Erythematosus and Deficiencies of Early Components of the Complement Classical Pathway. Frontiers in Immunology, 7, 55. https://doi.org/10.3389/fimmu.2016.00055

[2] Figueroa, J.E. and Densen, P. (1991) Infectious Diseases Associated with Complement Deficiencies. Clinical Microbiology Reviews, 4, 359-395.

https://doi.org/10.1128/CMR.4.3.359

[3] Picard, C., Al-Herz, W., Bousfiha, A., et al. (2015) Primary Immunodeficiency Diseases: an Update on the Classification from the International Union of Immunological Societies Expert Committee for Primary Immunodeficiency. Journal of Clinical Immunology, 35, 696. https://doi.org/10.1007/s10875-015-0201-1

[4] Li, N., Zhang, J., Liao, D., Yang, L., Wang, Y. and Hou, S. (2017) Association between $C 4, C 4 A$, and $C A B$ Copy Number Variations and Susceptibility to Autoimmune Diseases: A Meta-Analysis. Scientific Reports, 7, 42628.

https://doi.org/10.1038/srep42628

[5] Albin, S. and Cunningham-Rundles, C. (2014) An Update on the Use of Immunoglobulin for the Treatment of Immunodeficiency Disorders. Immunotherapy, 6, 1113-1126. https://doi.org/10.2217/imt.14.67

[6] Costa-Carvalho, B.T., Solé, D., Condino-Neto, A. and Rosário Filho, N. (2010) I Consenso Brasileirosobre o Uso de Imunoglobulina Humana em Pacientes com Imunodeficiências Primárias. Revista brasileira de alergia e imunopatologia, 33, 104-116.

[7] Liszewski, M.K. and Atkinson, J.P. (2015) Complement Regulator CD46: Genetic Variants and Disease Associations. Human Genomics, 9, 7.

https://doi.org/10.1186/s40246-015-0029-Z

[8] Turley, A.J., Gathmann, B., Bangs, C., Bradbury, M., Seneviratne, S., Gonzalez-Granado, L.I., et al. (2015) Spectrum and Management of Complement Immunodeficiencies (Excluding Hereditary Angioedema) across Europe. Journal of Clinical Immunology, 35, 199-205. https://doi.org/10.1007/s10875-015-0137-5

[9] Bing, D.H. and Alper, C.A. (1995) Complement in Health and Disease. In: Colvin, R.B., Bhan, A.K. and Mccluske, R.T., Eds., Diagnostic Immunopathology, 2nd Edition, Raven Press, New York, 85-94.

[10] Arason, G.J., Jorgensen, G.H. and Ludviksson, B.R. (2010) Primary Immunodeficiency and Autoimmunity: Lessons from Human Diseases. Scandinavian Journal of Immunology, 71, 317-328. https://doi.org/10.1111/j.1365-3083.2010.02386.X

[11] Cheng, H.-B., Chen, R.-Y., Wu, J.-P., et al. (2015) Complement C4 Induces Regulatory T Cells Differentiation through Dendritic Cell in Systemic Lupus Erythematosus. Cell \& Bioscience, 5, 73. https://doi.org/10.1186/s13578-015-0052-8

[12] Mayilyan, K.R. (2012) Complement Genetics, Deficiencies, and Disease Associations. Protein \& Cell, 3, 487-496. https://doi.org/10.1007/s13238-012-2924-6

[13] Robinson, D.P. and Klein, S.L. (2012) Pregnancy and Pregnancy-Associated Hor- 
mones Alter Immune Responses and Disease Pathogenesis. Hormones and Behavior, 62, 263-271. https://doi.org/10.1016/j.yhbeh.2012.02.023

[14] Fernandez-Perez, E.R., Salman, S., Pendem, S. and Farmer, C. (2005) Sepsis during Pregnancy. Critical Care Medicine, 33, S286-S293. ttps://doi.org/10.1097/01.CCM.0000182479.63108.CD

[15] Ward, R.G. and Walsh, M.S. (1991) Necrotizing Fasciitis: 10 Years' Experience in a District General Hospital. The British Journal of Surgery, 78, 488-489. https://doi.org/10.1002/bjs.1800780431

[16] Oud, L. and Watkins, P. (2014) Necrotizing Fasciitis Associated with Pregnancy: A Population-Based Cohort Study. Infectious Diseases and Therapy, 3, 307-320. https://doi.org/10.1007/s40121-014-0031-0

[17] Gallup, D.G., Freedman, M.A., Meguiar, R.V., Freedman, S.N. and Nolan, T.E. (2002) Necrotizing Fasciitis in Gynecologic and Obstetric Patients: A Surgical Emergency. American Journal of Obstetrics and Gynecology, 187, 305-311. https://doi.org/10.1067/mob.2002.126000

[18] Nikolaou, M., Zampakis, P., Vervita, V., et al. (2014) Necrotizing Fasciitis Complicating Pregnancy: A Case Report and Literature Review. Case Reports in Obstetrics and Gynecology, 2014, Article ID: 505410.

[19] Elliott, D.C., Kufera, J.A. and Myers, R.A.M. (1996) Necrotizing Soft Tissue Infections: Risk Factors for Mortality and Strategies for Management. Annals of Surgery, 224, 672-683. https://doi.org/10.1097/00000658-199611000-00011

[20] Brothers, T.E., Tagge, D.U., Stutley, J.E., Conway, W.F., Del Schutte Jr., H. and Byrne, T.K. (1998) Magnetic Resonance Imaging Differentiates between Necrotizing and Non-Necrotizing Fasciitis of the Lower Extremity. Journal of the American College of Surgeons, 187, 416-421. https://doi.org/10.1016/S1072-7515(98)00192-6

[21] Korhonen, K. (2000) Hyperbaric Oxygen Therapy in Acute Necrotizing Infections with a Special Reference to the Effects on Tissue Gas Tensions. Annales Chirurgiaeet Gynaecologiae, No. 214, 7-36.

[22] Brennan, V.M., Salomé-Bent, L. and Chapel, H.M. (2003) Immunology Nurses Study. Prospective Audit of Adverse Reactions Occurring in 459 Primary Antibody-Deficient Patients Receiving Intravenous Immunoglobulin. Clinical \& Experimental Immunology, 133, 247-251. https://doi.org/10.1046/j.1365-2249.2003.02199.x

[23] Kaul, R., McGeer, A., Norrby-Teglund, A., Kotb, M., Schwartz, B., O’Rourke, K., Talbot, J. and Low, D.E. (1999) The Canadian Streptococcal Study Group; Intravenous Immunoglobulin Therapy for Streptococcal Toxic Shock Syndrome-A Comparative Observational Study. Clinical Infectious Diseases, 28, 800-807. https://doi.org/10.1086/515199

[24] Kaul, R., et al. (1997) Population-Based Surveillance for Group A Streptococcal Necrotizing Fasciitis: Clinical Features, Prognostic Indicators, and Microbiologic Analysis of Seventy-Seven Cases. Ontario Group A Streptococcal Study. The American Journal of Medicine, 103, 18-24.

https://doi.org/10.1016/S0002-9343(97)00160-5 